\title{
Factors Related to Vulvovaginal Itching and Discharge among Iraqi Women
}

\author{
Dunya H. Al-Najjar'1 ${ }^{1}$ Zainab T. Al-Ogaili², Israa H. Al-Jerrah ${ }^{3}$ \\ ${ }^{1}$ M.B.CH.B., DGO, Al- Hindiya General Hospital, Karbala Health Director, ${ }^{2}$ M.B.CH.B., DGO, Baghdad Health \\ Director, ${ }^{3}$ M.B.CH.B., M.Sc. Microbiology and Immunology, Al- Hindiya General Hospital, Karbala Health
}

Director, Karbala, Iraq

\begin{abstract}
Background: Vaginal infections are considered global health problems for women in child-bearing ages. At the time in which various pathogenic organisms are responsible for those infections, many risk factors may also be attributed for such infections, including sexual activity and partner, contraceptive use, personal hygiene level, female estrogen level and its effect on vaginal mucosa, medical history, and many other factors. However, the disease by itself is not considered an exclusive sexually transmitted infection.

Objective: The goal of the Study is to identify the causes and factors related to the symptoms of vulvovaginal itching and/or vaginal discharge.

Methodology: A cross sectional study conducted on 93 females (16 pregnant $\& 77$ non pregnants females) attending the private clinics in Al-Hindiya district (Karbala governorate) from May 2019 to September 2019. Ethically, patients' information was treated with confidentiality and privacy throughout data collection and analysis. Tow swabs had been taken from each patient (vaginal and cervical) and sent to a private lab for direct microscopic examination and culturing on appropriate media. Statistically, the quantitative variables were analyzed using non-parametric t-test and the qualitative variables were analyzed using Chi-square test.

Results: Fortunately, the age of all participants was within child-bearing age ranging 15 to 42 with a mean of $28.0 \pm 6.9$ years. There was no significant association of pregnancy with vaginal infections as noticed in this study, while diabetes mellitus (DM) was found to be significantly related to the presence of infection (P-value $=0.003$ ). Similarly, intrauterine contraceptive device (IUCD) usage was also found to be significantly related to the occurrence of infection $(\mathrm{P}$-value $=0.040)$. On the other, thelab study showed that E. coli is the most common pathogen to be expected in women complaining of vulvovaginal itching and/or vaginal discharge with monilia being the second.

Conclusions: DM and IUCD usage had their burdens on the vaginal cavity causing increasing rate of vaginal symptoms as proven significantly. Simultaneously, the cultivation results revealed that E.coli is the causative agent for of vulvovaginal itching and/or vaginal discharge at the level of our females, a point meriting further evaluation.
\end{abstract}

Keywords: Vulvovaginal itching, vaginal discharge, vaginal and cervical swabs, E.coli.

\section{Introduction}

Vaginal infections are considered some of the most important health problems for women all around the world $^{[1]}$. Various pathogenic organisms are responsible for those infections, including bacterial vaginosis, candidiasis, and trichomoniasis ${ }^{[2]}$. Bacterial vaginosis is considered a clinical syndrome of multiple pathogens that results from replacement of Lactobacilli species (which are normally found in vagina) with anaerobic bacteria $^{[3]}$.

Patients are usually presented with symptoms of itching or burning sensation in the vulva or vagina, 
abnormal vaginal discharge, or irritation and discomfort. Some of the infections are reported to be present with minimal symptoms ${ }^{[4]}$. Many risk factors were suggested for bacterial vaginosis, including sexual activity and contraceptive use, although the disease itself is not considered an exclusive sexually transmitted infection ${ }^{[5]}$.

Several complications may occur as a result of vaginal infections, such as pelvic inflammatory disease (PID) that may lead to further consequences such as infertility, ectopic pregnancy, preterm labour, and low birth weight ${ }^{[6,7]}$.

Diagnosis is usually achieved by clinical examination alone or with the aid of laboratory diagnosis. Classically using direct microscopic exam or cultivation for vaginal swabs ${ }^{[8]}$. The study aims to identify the causes and factors related to the symptoms of vulvovaginal itching and discharge.

\section{Patients and Method}

A cross sectional study conducted on a randomly selected 93 females (16 pregnant \& 77 non pregnants females fulfilled all inclusion and exclusion criteria) attending the private clinics in Al-Hindiya district (Karbala governorate) from May 2019 to September 2019. Ethically verbal informed consent was obtained from all the patients who were enrolled in data collection after explaining the objectives of the study. Patients' information was treated with confidentiality and privacy throughout data collection and analysis. Also, patient's permission had been taken to publish the study.

Data including demography, gravida/para/abortus (GPA), medical history, previous vaginal infections, contraceptive method as well as clinical examination were collected using specially designed format. Tow swabs had been taken from each patient (high vaginal and endocervical) and sent to a private lab where, after processing according to the standard laboratory procedures, a direct microscopic examination done for the vaginal swabs and culturing on appropriate media for the cervical swabs.

The direct microscopic examination done by wet preparation of vaginal swabs, where we looked for epithelial cells, bacteria adherent to epithelial cells, Lactobacilli as their presence in suitable number indicate healthy vagina, and also for polymorphonuclear (PMN) cells. Sometimes, motile bacteria could be seen, giving a correct diagnosis of infecting micro-organism.
Unfortunately, the direct examination of vaginal swabs was inconclusive for most of the patients, which may be due to very superficial swabbing or lab technical errors.

The cervical swabs had processed and each one cultivated on two separated agars, MacConkey, and blood agar, then incubated at $37 \mathrm{c}$ for 24 hour in an aerobic environment. The growth of colonies revealed 1-2 days after cultivation. The plates which had demonstrated bacterial colonies, sent for identification of the type of bacteria using the VITEK 2 compact (automated microbiology system of bioMerieux).

Principles of VITEK2 system: This system utilizes growth-based technology and accommodates a colorimetric reagent cards that are incubated and interpreted automatically. There are four reagent cards that currently used for the identification of different organisms:

1. GN-Gram negative fermenting and non-fermenting bacilli

2. GP -Gram positive cocci and non-spore forming bacilli

3. YST -yeast and yeast-like organisms

4. BCL-Gram positive spore forming bacilli

The reagent cards have 64 wells that can each contain an individual substrate. Substrates measure the various metabolic activities such as acidification, alkalinization, enzyme hydrolysis, and growth in the presence of inhibitory substances. An optically clear film present on both sides of the card allows for appropriate level of oxygen transmission while maintaining a sealed vessel that prevents contact with organism-substrate admixture. Each card has bar code, lot number, expiration date, and a unique identifier.

Statistical Package for Social Sciences $\left(\operatorname{SPSS}^{\circledR}\right)$ Software version 23.0 was used to perform statistical analysis. Qualitative data are presented as number and percentage, while continuous numerical data are presented as mean \pm standard deviation. Comparisons between study variables were carried out using Chisquare test for categorical data and Student's t-test for numerical data. P-value of $<0.05$ was considered statistically significant.

\section{Results}

Demographic characteristics of patients: This 
study enrolled a total of 93 patients complaining of vulvovaginal itching and/or vaginal discharge. The patients' age ranged from 15 to 42 years, with a mean of $28.0 \pm 6.9$ years (Figure 1).
In regards to the gravida/para/abortus (GPA) system; the median gravida score of study participants was 3 (G3), median para score was 3 (P3), and median abortus score was 0 (A0). However, these information had not represented by a figure.

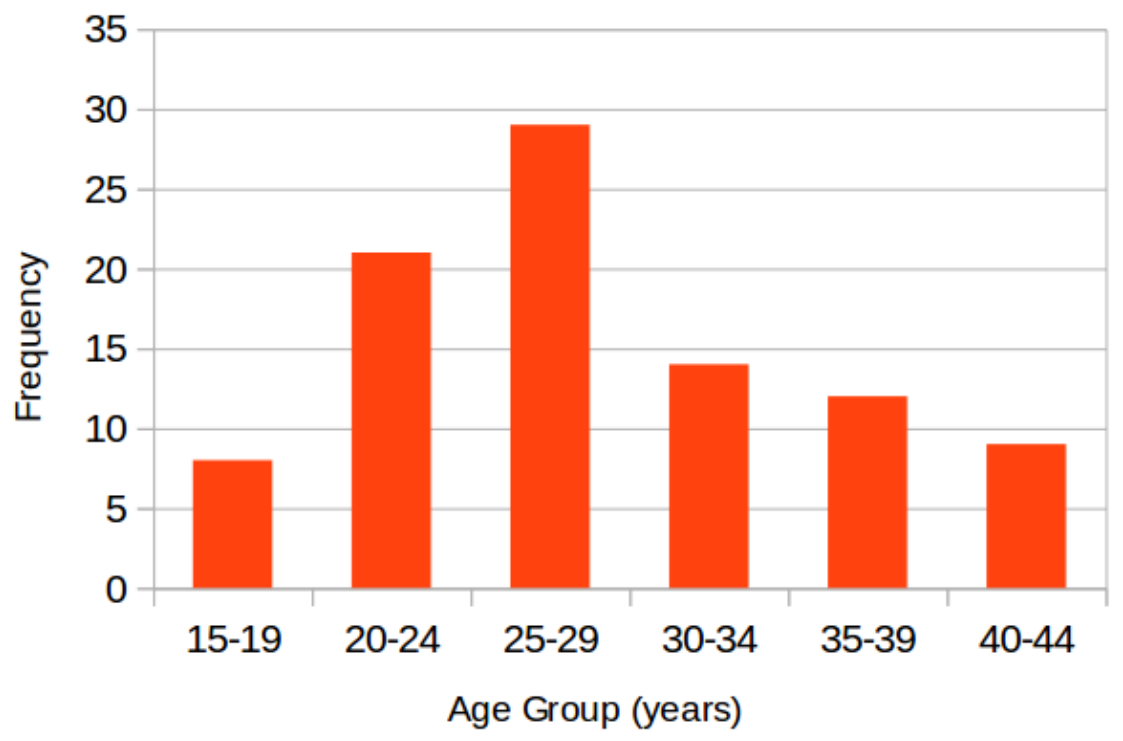

Figure 1: Age group distribution of the study participants

Association of DM, pregnancy, and IUCD usage with vaginal infections: To assess the role of DM, pregnancy, and IUCD usage on the occurrence of infection; those variables were compared to the presence of infection using chi-square test.

1. DM was found to have significant relationship with the presence of infection among patients in the study (P-value $=0.003$ ). More than $83 \%$ of diabetic patients had infection, while approximately $38 \%$ of non-diabetic patients had infection. The odds ratio for diabetic patients to have an infection was calculated to be 3.7 .
2. Association of pregnancy with vaginal infections had shown no statistically significant relationship, with chi-square $=0.34$, d.f. $=1$, P-value $=0.560$, as presented in Table 1.

3. Fisher Exacts test was used to assess the association of IUCD use with vaginal infections and there was a statistically significant relationship between IUCD usage and presence of infection, Fisher Exacts P-value $=0.040$, as described in the table below. Odds ratio was found to be 2.68. Infection was present in about $77 \%$ of women who used IUCD, while it was present in only $40 \%$ of women not using IUCD.

Table 1: Association of DM, pregnancy, and IUCD usage with vaginal infections:

\begin{tabular}{|c|c|c|c|c|}
\hline \multirow{2}{*}{ Patient history of DM } & \multicolumn{3}{|c|}{ Presence of bacterial/fungal growth No. (\%) } & \multirow{2}{*}{ P-value } \\
\hline & Present & Absent & Total & \\
\hline Diabetic & $10(83.33 \%)$ & $2(16.67 \%)$ & $12(100 \%)$ & \multirow{3}{*}{0.003} \\
\hline Non-diabetic & $31(38.27 \%)$ & $50(61.73 \%)$ & $81(100 \%)$ & \\
\hline Total & $41(44.09 \%)$ & $52(55.91 \%)$ & $93(100 \%)$ & \\
\hline
\end{tabular}




\begin{tabular}{|c|c|c|c|c|}
\hline \multirow{2}{*}{ Pregnancy } & \multicolumn{3}{|c|}{ Presence of bacterial/fungal growth No. (\%) } & \multirow{2}{*}{ P-value } \\
\hline & Present & Absent & Total & \\
\hline Pregnant & $6(37.50 \%)$ & $10(62.50 \%)$ & $16(100 \%)$ & \multirow{3}{*}{0.560} \\
\hline Non-pregnant & $35(45.45 \%)$ & $42(54.55 \%)$ & $77(100 \%)$ & \\
\hline Total & $41(44.09 \%)$ & $52(55.91 \%)$ & $93(100 \%)$ & \\
\hline \multicolumn{5}{|c|}{ Chi-square $=0.34$, d.f. $=1, \mathrm{P}$-value $=0.560$} \\
\hline \multirow{2}{*}{ IUCD usage } & \multicolumn{3}{|c|}{ Presence of bacterial/fungal growth No. (\%) } & \multirow{2}{*}{ P-value } \\
\hline & Present & Absent & Total & \\
\hline Used & $7(77.78 \%)$ & $2(22.22 \%)$ & $9(100 \%)$ & \multirow{3}{*}{0.040} \\
\hline Not used & $34(40.48 \%)$ & $50(59.52 \%)$ & $84(100 \%)$ & \\
\hline Total & $41(44.09 \%)$ & $52(55.91 \%)$ & $93(100 \%)$ & \\
\hline
\end{tabular}

Association of patient's age with vaginal infections: No significant relationship was found between presence of infection and the age of the patient, with a Student's t-test $=0.40$, d.f. $=91$, P-value $=0.687$, as detailed in Table 2 .

Table 2: Association of patient's age with vaginal infections:

\begin{tabular}{|c|c|c|c|c|}
\hline \multirow{2}{*}{$\begin{array}{l}\text { Presence of bacterial/fungal } \\
\text { growth }\end{array}$} & \multicolumn{3}{|c|}{ Age (years) } & \multirow{2}{*}{ P-value } \\
\hline & Mean \pm SD & Median & Range & \\
\hline Present $(n=41)$ & $27.7 \pm 6.7$ & 26.0 & $18.0-41.0$ & \multirow{3}{*}{0.687} \\
\hline Absent $(n=52)$ & $28.3 \pm 7.2$ & 28.0 & $15.0-42.0$ & \\
\hline Total $(n=67)$ & $28.0 \pm 6.9$ & 27.0 & $15.0-42.0$ & \\
\hline
\end{tabular}

Pathogenic agents causing vaginal infections: infection was monilia, comprising $10 \%$ of the study The most common pathogen found among study population, half of which was concomitant with E. coli participants was $E$. coli, with a percentage of more than infection. $32 \%$, as illustrated in Figure (2). The next most common

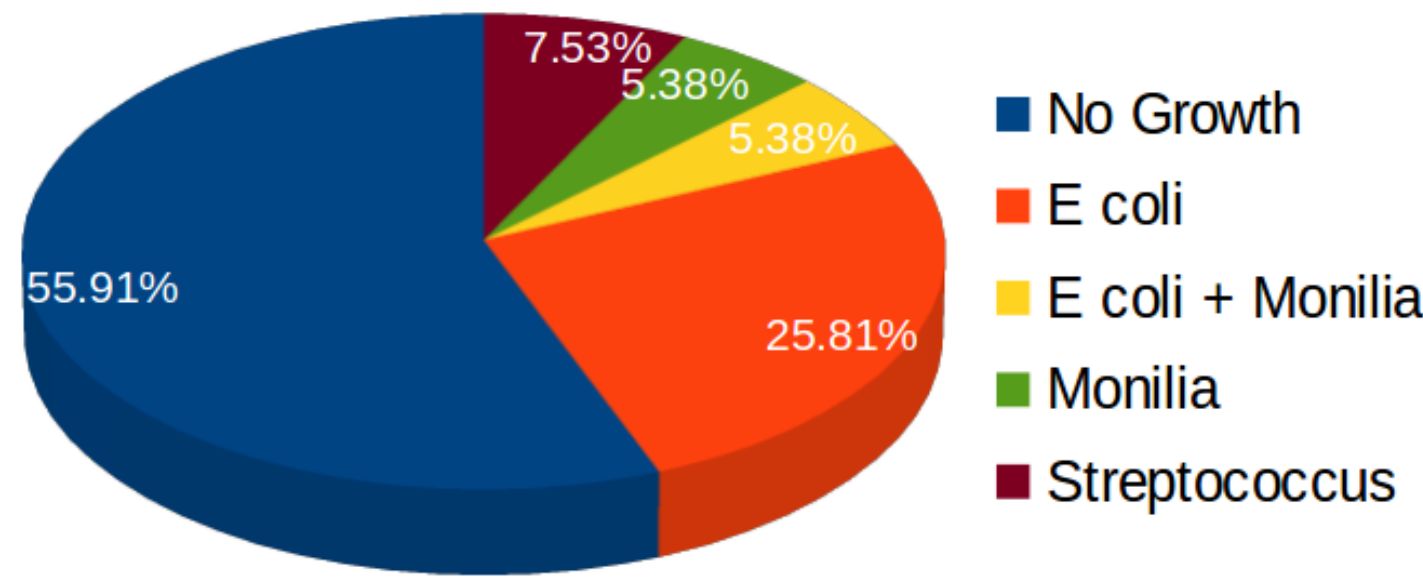

Figure 2: Types of Pathogenic agents causing vaginal infections: 
Association of DM, pregnancy, and IUCD usage with pathogenic agents: To further extend the analysis, each pathogenic organism was compared to DM, pregnancy, and IUCD usage.

1. DM was found to have significant relationship to $E$. coli infection, but not to the other types of infection (Table 3).
2. On the other hand, neither pregnancy nor IUCD use were found to have statistically significant relationship with a particular type of infection (pathogenic agent).

Table 3:Association of DM, pregnancy, and IUCD usage with pathogenic agents:

\begin{tabular}{|c|c|c|c|}
\hline \multirow{2}{*}{ Patient history of DM } & \multicolumn{3}{|c|}{ Type of growth No. (\%) } \\
\hline & E. coli & Streptococcus & Monilia \\
\hline Diabetic $(n=12)$ & $10(83.33 \%)$ & $0(0 \%)$ & $0(0 \%)$ \\
\hline Non-diabetic $(\mathrm{n}=81)$ & $19(23.46 \%)$ & $7(8.64 \%)$ & $10(12.35 \%)$ \\
\hline Total $(n=93)$ & $29(31.18 \%)$ & $7(7.53 \%)$ & $10(10.75 \%)$ \\
\hline P-value & $<0.001$ & 0.589 & 0.350 \\
\hline \multirow{2}{*}{ Pregnancy status } & \multicolumn{3}{|c|}{ Type of growth No. (\%) } \\
\hline & E. coli & Streptococcus & Monilia \\
\hline Pregnant(n=16) & $5(31.25 \%)$ & $1(6.25 \%)$ & $1(6.25 \%)$ \\
\hline Non-pregnant(n=77) & $24(31.17 \%)$ & $6(7.79 \%)$ & $9(11.69 \%)$ \\
\hline Total $(n=93)$ & $29(31.18 \%)$ & $7(7.53 \%)$ & $10(10.75 \%)$ \\
\hline P-value & 0.995 & 0.832 & 0.523 \\
\hline \multirow{2}{*}{ IUCD usage } & \multicolumn{3}{|c|}{ Type of growth No. (\%) } \\
\hline & E. coli & Streptococcus & Monilia \\
\hline Used $(n=9)$ & $5(55.56 \%)$ & $2(22.22 \%)$ & $2(22.22 \%)$ \\
\hline Not used $(n=84)$ & $24(28.57 \%)$ & $5(5.95 \%)$ & $8(9.52 \%)$ \\
\hline Total $(n=93)$ & $29(31.18 \%)$ & $7(7.53 \%)$ & $10(10.75 \%)$ \\
\hline P-value & 0.131 & 0.326 & 0.248 \\
\hline
\end{tabular}

\section{Discussion}

Women included within this study were aged between 15-42 years, which roughly represent the reproductive age in women. The highest proportion of patients was within the age group 25-29years, representing the peak age of sexual activity and the sequel of vaginal symptoms.

Given the increasing rate in vaginal infection among females, many interfering factors to be verified due to their impact in causing vaginal symptoms including, patient's general health, medical history, sexual activity and sexual partner, pregnancy, type of contraceptive used,, PH, change in hormonal balance, poor vaginal hygiene or over use of vaginal douches or perfumed spray, antibiotic and immunosuppressive drugs use, underwear quality, degree of moisture, concomitant infections, frequency of changing pads during period, the way of washing in toilet, and others.

Statistical analysis of patients' data had demonstrated the following important points:

1. DM has a significant impact in causing infection among young women, with odds ratio of 3.7. This means that diabetic women are approximately 4 times more likely to develop vulvovaginal infection than non-diabetic women. This finding is consistent with the finding by Nowakowska et al. in their case- 
control study conducted on 251 women, in which they concluded that risk for vaginal infection is more than 4 times higher in women with DM compared to control group ${ }^{[9]}$.

2. Although all of us agree that pregnancy lowers the immune response of pregnant female making her more susceptible to infection, this study reflects a non-significant effect of pregnancy on the occurrence of vulvovaginal infection. Proportion of pregnant females who had positive result for infection was found to be $37.5 \%$. This percentage was closely similar to the finding by Mengistie et al. in their study conducted on 252 pregnant females from 2011 to 2012, which found that $36.7 \%$ of symptomatic pregnant women included in the study had positive microbiological diagnosis ${ }^{[10]}$.

3. At the time in which IUCD has important role in preventing pregnancy, noticeable side effects on the womb had been reported ranging between heavy menses to recurrent vaginal infection, and to be tolerated by the users. Here it was found that more than $77 \%$ of women who used IUCD had positive result for infection, compared to $40 \%$ of women who did not use IUCD. This finding is consistent with the finding by Jabuk S. in her study conducted in Al-Hilla city from 2012 to 2013, which included 50 women who used IUCD, which concluded that IUCD use significantly increased the risk for bacterial vaginosis compared to other contraceptive method ${ }^{[11]}$.

4. An interesting observation in the study was the significant relationship between diabetes and specific type of microorganisms, namely E. coli., where we always thinks that Candida albicans is the dominant in DM patients. This could reflect the opportunistic nature for this microorganism which is important to note in patients with $\mathrm{DM}^{[12]}$. However, further evaluation is needed to elucidate their opportunity. The second important point is that $E$. coli. is normal inhabitant of rectum and their transport to vagina may indicate poor hygiene with retrograde (recto-vaginal) washing, where changing $\mathrm{PH}$ and effect of female hormones may alter these bacteria into pathogenic type causing infection.

\section{Conclusions}

This study has demonstrated that:vaginal infections may occur at any point of reproductive age of patients, where some factors may increase their susceptibility, and the most common infection to be expected in women complaining of vulvovaginal itching and/or discharge is E. coli. A significant relationship between IUCD usage and DM in occurrence of infection, Vaginal infections particularly with E. coli. are closely related to DM.

Financial Disclosure: There is no financial disclosure.

Conflict of Interest: None to declare.

Ethical Clearance: All experimental protocols were approved under the Al- Hindiya general hospital and all experiments were carried out in accordance with approved guidelines.

\section{References}

1. Go V, Quan V, Celentano D, Moulton L, Zenilman M. Prevalence and risk factors for reproductive tract infections among women in rural Vietnam. The Southeast Asian Journal of Tropical Medicine and Public Health. 2006;37(1):185-89.

2. Wang H, Huang $\mathrm{Zh}, \mathrm{Wu} \mathrm{Zh}$, Qi X, Lin D. An epidemiological study on vaginitis in 6,150 women of reproductive age in Shanghai. NewMicrobiologica. 2017;40(2):113-18.

3. Workowski K, Bolan G, Division of STD Prevention/CDC. Sexually Transmitted Diseases Treatment Guidelines, 2015.Morbidity and Mortality Weekly Report. 2015;64(3).

4. Adeyeba O, Adeoye M, Adesiji Y. Bacteriological and Parasitological Assessment of Vaginitis in Pregnant Women in Iseyin, Oyo State, Nigeria. African Journal of Clinical \& Experimental Microbiology. 2003;4(2):116-26.

5. Trabert B, Misra D. Risk factors for bacterial vaginosis during pregnancy among African American women. American Journal of Obstetrics \& Gynecology. 2007;197(5):477.e1-477.e8.

6. Mulu W, Yimer M, Zenebe Y, Abera B. Common causes of vaginal infections and antibiotic susceptibility of aerobic bacterial isolates in women of reproductive age attending at Felegehiwot referral Hospital, Ethiopia: a cross sectional study. BMC Women's Health. 2015;15(42):1-9.

7. Filho D, Diniz C, Silva V. Bacterial vaginosis: clinical, epidemiologic and microbiological features. HU Revista. 2010;36(3):223-30. 
8. Money D. The laboratory diagnosis of bacterial vaginosis.Canadian Journal of Infectious Diseases and Medical Microbiology.2005;16(2):77-79.

9. Nowakowska D, Kurnatowska A, Stray-Pefersen B, Wilczynski J. Prevalence of fungi in the vagina, rectum and oral cavity in pregnant diabetic women: relation to gestational age and symptoms. ActaObstetriciaetGynecologicaScandinavica. 2004;83:251-56.

10. Mengistie Z, Woldeamanuel Y, Asrat D, Adera A. Prevalence of bacterial vaginosis among pregnant women attending antenatal care in TikurAnbessa
University Hospital, Addis Ababa, Ethiopia. BMC Research Notes. 2014;7(822):1-5.

11. Jabuk S. Prevalence of aerobic bacterial vaginosis among Intrauterine Contraceptive Device users women in Hilla city. Journal of Babylon University/ Pure and Applied Sciences.2014;22(9):2424-31.

12. Razzak M, Al-Charrakh A, Al-Greitty B. Relationship between lactobacilli and opportunistic bacterial pathogens associated with vaginitis. North American Journal of Medical Sciences. 2011;3(4):185-92. 